\title{
Reversible pulmonary trunk banding. VI: Glucose-6-phosphate dehydrogenase activity in rapid ventricular hypertrophy in young goats
}

\author{
Renato S. Assad, MD, PhD, ${ }^{a}$ Fernando A. Atik, MD, ${ }^{\mathrm{b}}$ Fernanda S. Oliveira, ${ }^{\mathrm{a}}$

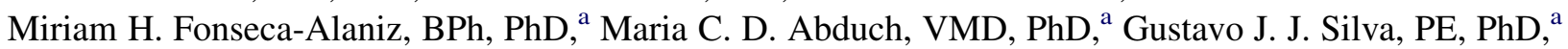 \\ Gustavo G. Favaro, MD, ${ }^{\mathrm{a}}$ Jose E. Krieger, $\mathrm{MD}, \mathrm{PhD},{ }^{\mathrm{a}}$ and Noedir A. G. Stolf, $\mathrm{MD}, \mathrm{PhD}^{\mathrm{a}}$
}

Objective: Increased myocardial glucose-6-phosphate dehydrogenase (G6PD) activity occurs in heart failure. This study compared G6PD activity in 2 protocols of right ventricle (RV) systolic overload in young goats.

Methods: Twenty-seven goats were separated into 3 groups: sham (no overload), continuous (continuous sys-
tolic overload), and intermittent (four 12-hour periods of systolic overload paired with a 12-hour resting period).
During a 96-hour protocol, systolic overload was adjusted to achieve a 0.7 RV/aortic pressure ratio. Echocardio-
graphic and hemodynamic evaluations were performed before and after systolic overload every day postopera-
tively. After the study period, the animals were humanely killed for morphologic and G6PD tissue activity
assessment.

Results: A 92.1\% and 46.5\% increase occurred in RV and septal mass, respectively, in the intermittent group compared with the sham group; continuous systolic overload resulted in a $37.2 \%$ increase in septal mass. A worsening RV myocardial performance index occurred in the continuous group at 72 hours and 96 hours, compared with the sham $(P<.039)$ and intermittent groups at the end of the protocol $(P<.001)$. Compared with the sham group, RV G6PD activity was elevated $130.1 \%$ in the continuous group $(P=.012)$ and $39.8 \%$ in the intermittent group $(P=.764)$.

Conclusions: Continuous systolic overload for ventricle retraining causes RV dysfunction and upregulation of myocardial G6PD activity, which can elevate levels of free radicals by NADPH oxidase, an important mechanism in the pathophysiology of heart failure. Intermittent systolic overload promotes a more efficient RV hypertrophy, with better preservation of myocardial performance and and less exposure to hypertrophic triggers.

(J Thorac Cardiovasc Surg 2011;142:1108-13)

Supplemental material is available online.

Traditional pulmonary artery banding (PAB) aimed at ventricular retraining causes an abrupt and fixed systolic overload. Although clinical studies have proved that PAB induces myocardial hypertrophy, it is frequently preceded by ventricular dysfunction. Therefore, an adaptation period

\footnotetext{
From the Heart Institute, ${ }^{a}$ University of São Paulo Medical School, São Paulo, Brazil; and Instituto de Cardiologia do Distrito Federal, ${ }^{\mathrm{b}}$ Brasilia, Brazil.

Supported by FAPESP (São Paulo State Foundation for Research Support) grant no. 2006/50831-2. Banding devices were provided by SILIMED Inc (Rio de Janeiro, Brazil).

Disclosures: Authors have nothing to disclose with regard to commercial support.

Read at the 91st Annual Meeting of The American Association for Thoracic Surgery, Philadelphia, Pennsylvania, May 7-11, 2011.

Received for publication May 3, 2011; revisions received June 29, 2011; accepted for publication Aug 4, 2011; available ahead of print Sept 12, 2011

Address for reprints: Renato S. Assad, MD, PhD, Heart Institute University of São Paulo Medical School, Division of Surgical Research, Ave Dr Eneas C. Aguiar, 44, São Paulo, SP-Brazil 05403-000 (E-mail: rsassad@cardiol.br).

0022-5223/\$36.00

Copyright (C) 2011 by The American Association for Thoracic Surgery doi: $10.1016 /$ j.jtcvs.2011.08.007
}

with inotropic support is generally required. Most important, previous studies have demonstrated myocardial edema and necrosis in hearts that experience abrupt systolic overload, followed by late ventricular failure. ${ }^{1}$

However, it is essential to understand the molecular mechanisms involved in PAB-induced myocardial hypertrophy to establish training protocols that lead to a desirable "physiologic hypertrophy" versus a deleterious "pathologic hypertrophy." Because a known shift occurs in energy substrate use in favor of glucose in pathologic conditions, energy metabolism might be altered in PAB ventricular retraining protocols. ${ }^{2}$ In addition, recent experimental studies have linked an unbalanced oxidative and reductive process to a variety of diseases, such as atherosclerosis and heart failure. ${ }^{3}$

Glucose 6-phosphate dehydrogenase (G6PD), the ratelimiting enzyme that commits glucose to the pentose phosphate pathway, is mainly responsible for the generation of nicotinamide adenine dinucleotide phosphate (NADPH) and ribose 5-phosphate, an essential precursor of the de novo synthesis of RNA and DNA. G6PD-derived NADPH, a cofactor for glutathione and thioredoxin reductase, preserves reducing potentials and protects the cell from 


$$
\begin{array}{ll}
\text { Abbreviations and Acronyms } \\
\begin{aligned}
\text { G6P } & \text { glucose-6-phosphate } \\
\mathrm{G} 6 \mathrm{PD} & =\text { glucose } 6 \text {-phosphate dehydrogenase } \\
\mathrm{LV} & =\text { left ventricle } \\
\mathrm{NADP}^{+}= & \text {oxidized nicotinamide adenine } \\
& \text { dinucleotide phosphate } \\
\mathrm{NADPH}= & \text { nicotinamide adenine dinucleotide } \\
& \text { phosphate } \\
\mathrm{PA} & =\text { pulmonary artery } \\
\mathrm{PAB} & =\text { pulmonary artery banding } \\
\mathrm{RV} & =\text { right ventricle }
\end{aligned}
\end{array}
$$

oxidative stress in normal conditions. ${ }^{4}$ In human diseases, G6PD can be either activated or inhibited; however, evidence has emerged that the overexpression and activation of G6PD enhances NADPH oxidase-derived superoxide generation and increases oxidative stress in diseases like diabetes, heart failure, and hypertension. ${ }^{5}$

In regard to rapid ventricular training, it would be of great interest to study myocardial energy metabolism in response to different cardiac hypertrophy models and its relationship to heart function. ${ }^{6}$ The main objective of this study was to compare the G6PD activity in 2 right ventricle (RV) training protocols through an adjustable PAB system.

\section{METHODS}

Twenty-seven young goats, aged between 30 and 60 days and of comparable weight $(P=.38)$, were split into 3 groups: sham $(\mathrm{n}=7$; weight, $11.93 \pm 2.67 \mathrm{~kg})$, continuous ( $\mathrm{n}=9$; weight, $10.74 \pm 2.62 \mathrm{~kg})$, and intermittent $(\mathrm{n}=11$; weight, $10.25 \pm 2.20 \mathrm{~kg}$ ). All animals received humane care in compliance with the guidelines established by the Brazilian regulations for animal experimentation. The protocol was reviewed and approved by the Ethics Committee for Research Protocols at the University of São Paulo Medical School.

\section{Surgical Procedure}

All operations were performed with the goats under general anesthesia (pentobarbital sodium $5 \mathrm{mg} / \mathrm{kg}$ intravenously and ketamine $20 \mathrm{mg} / \mathrm{kg}$ intramuscularly) and through a left lateral thoracotomy. The lung was retracted laterally to allow exposure of the right ventricular outflow tract, pulmonary trunk, and descending aorta. A 17-gauge heparinized catheter was inserted into each of these structures, and its corresponding pressures were measured at specific time intervals during the entire study. The adjustable PAB system (SILIMED; Silicone e Instrumental Médico-Cirúrgico e Hospitalar Ltda, Rio de Janeiro, Brazil) was implanted just beyond the pulmonary valve and sutured at the adventitia of the pulmonary trunk, as previously described (Figure E1). ${ }^{7}$ Antibiotics (cefazolin $500 \mathrm{mg}$ and gentamicin $40 \mathrm{mg}$ ) were administered daily during the study, as were digoxin $(0.04 \mathrm{mg} / \mathrm{kg})$ and subcutaneous heparin $(5000 \mathrm{U})$.

\section{Training Protocol}

RV systolic overload was initiated 72 hours postoperatively. Baseline hemodynamic data (RV, pulmonary artery [PA], and aortic pressures) were collected in a conscious, immobilized animal with the adjustable banding system deflated. Blood pressure measurements were obtained through computer software (ACQknowledge 3.01; Biopac Systems, Inc, Goleta, Calif). Then, the banding system was adjusted to achieve an RV/ aortic pressure ratio of 0.7 , limited by a $10 \%$ drop in systolic blood pressure. Adjustments were made just once, every morning throughout the protocol, by percutaneous injection of saline solution with a 3-mL syringe, under sterile conditions. That rule was violated in case of the latter occurring or if there were agitation, dyspnea, arrhythmia, or a combination of these. The banding system was then deflated up to a tolerable point.

\section{Continuous Group Protocol}

The animals remained with continuous systolic overload for 96 hours, with daily assessment to keep the RV/aortic pressure ratio at 0.7 . Hemodynamic data were collected once a day (mornings) during PAB readjustments.

\section{Intermittent Group Protocol}

The animals underwent 4 daytime periods of RV 12-hour systolic overload, alternating with a 12 -hour nighttime resting period. Hemodynamic data were collected twice a day (every 12 hours) during PAB readjustments.

\section{Sham Group Protocol}

The PAB system was maintained deflated during the entire protocol. Hemodynamic data were collected daily (mornings).

\section{Echocardiography}

A single experienced observer conducted the echocardiographic examination with the animals under light sedation (ketamine $15 \mathrm{mg}$ intramuscularly) approximately 120 hours before the beginning of the protocol and daily thereafter until the end of the protocol. Image acquisition was obtained through 7.5-MHz and 2.5-MHz multifrequency transducers (Acuson Cypress Echocardiology System, Siemens, Erlagen, Germany). The following echocardiographic parameters were studied: left ventricle (LV), $\mathrm{RV}$, and septal wall thicknesses, RV end-diastolic volume, and myocardial performance index.

\section{Morphology}

Animals were humanely killed after 96 hours of the study protocol. Cardiac samples were drawn from the RV, LV, and ventricular septum just before cardiac arrest. These samples were immediately frozen at $-80^{\circ} \mathrm{C}$ (Forma Scientific Inc, Marietta, Ohio) to be subsequently analyzed for G6PD activity. The pericardial fat, both atria, and semilunar valves were dissected from the heart; RV, LV, and ventricular septum were separated by the Fulton technique, individually weighed (METTLER AE-200; Mettler-Toledo AG, Greifensee, Switzerland), and indexed to each animal's body weight. ${ }^{8}$

Water content was obtained individually in each cardiac chamber by subtracting the collected sample weight at autopsy from the weight of the dehydrated chamber $\left(70\right.$ hours at $\left.60^{\circ} \mathrm{C}\right)$. Values were obtained as a percentage of weight change.

\section{G6PD Activity}

Tissue samples were homogenized in extraction buffer (proportion 1:5 weight/volume). The material was stored in ice and homogenized for 30 seconds using Polytron (PT 3100; Kinematica AG, Littau-Lucerne, Switzerland) at maximum speed and centrifuging $\left(15 \mathrm{~kg}, 15\right.$ minutes, $\left.4^{\circ} \mathrm{C}\right)$ to separate from cell remnants. Enzymatic activity was analyzed using the supernatant of the last centrifugation. Proteins were quantified with the protein assay kit BCA (PIERCE Biotechnology, Rockford, Ill). Results are expressed as nmol min $\mathrm{mg}^{-1}$ of protein. The extraction buffer for G6PD contained Tris- $\mathrm{HCl}(50 \mathrm{mmol} / \mathrm{L})$ and ethylenediaminetetraacetic acid $(1 \mathrm{mmol} / \mathrm{L})$, with a $\mathrm{pH}$ of 8 . The assay buffer $(270 \mu \mathrm{L} / \mathrm{sample})$ was Tris- $\mathrm{HCl}(8.6 \mathrm{mmol} / \mathrm{L}), \mathrm{MgCl}_{2}(6.9 \mathrm{mmol} / \mathrm{L})$, (oxidized nicotinamide 
adenine dinucleotide phosphate (NADP ${ }^{+} ; 0.4 \mathrm{mmol} / \mathrm{L}$ ), and Triton $\mathrm{X}-100$ $0.05 \%$ (volume/volume), with a $\mathrm{pH}$ of 7.6. The reaction was initiated by adding $15 \mu \mathrm{L}$ of glucose-6-phosphate (G6P; $1.2 \mathrm{mmol} / \mathrm{L}$ ) to the enzymatic extract ( $15 \mu \mathrm{L}$ of sample) for 10 minutes at $25^{\circ} \mathrm{C}$. The absorbance was monitored at $340 \mathrm{~nm}$, the extinction coefficient being 6.22 for that particular wavelength. The biochemical reaction is based on the glucose phosphorylation into G6P, and posterior formation of 6-phosphogluconate by the action of G6PD. The G6PD activity was indirectly determined by the total production of NADPH through the pentose phosphate pathway. ${ }^{9} \mathrm{RV}$ and ventricular septum values were indexed to each animal's LV value.

\section{Statistical Analysis}

Values are expressed as means and standard deviation. Comparison of variables was performed with 2-way analysis of variance, except for body weight, G6PD activity, wall masses, and water content, which were performed with 1-way analysis of variance. Both analyses were followed by the Bonferroni post hoc test. The level of significance used was $5 \%$. Statistical analysis was performed using GraphPad Prism version 4 software (GraphPad Prism, La Jolla, Calif) and SigmaStat 3.11.0 for Windows (Systat Software, Inc, Chicago, Ill).

\section{RESULTS}

\section{Hemodynamic Variables}

Systolic RV-PA gradient increased in both study groups $(P<.001)$ and in every time period $(P<.001)$. There was no significant difference of banding gradients between the 2 study groups on each day of the protocol, being both submitted to the same magnitude of systolic overload stimulus (Table E1). Likewise, both study groups showed an increased RV-aorta systolic pressure ratio over time (Figure E2) compared with its respective baseline and with that in the sham group at the same time $(P<.05)$. There were peak systolic gradients in the intermittent group, alternating with 12-hour rest periods when they were similar to those in the sham group (Figure 1, $A$ ). The small RV-PA gradient $(3.83 \pm 1.33 \mathrm{~mm} \mathrm{Hg}$ to $5.50 \pm 1.38 \mathrm{~mm} \mathrm{Hg})$ observed in the sham group is considered physiologic. To quantify the exposure to systolic overload, we determined the area under the RV-PA gradient curve (Figure 1, B). The continuous group was the group most exposed to systolic overload $(P<.05)$.

\section{Echocardiographic Variables}

RV wall thickness was thinner than septal and LV thicknesses in all animals preoperatively (group factor, $P=.663$; heart wall factor, $P<.001$ ). There was a marked increase in $\mathrm{RV}$ wall thickness after 48 hours in the intermittent group and after 72 hours in the continuous group compared with the baseline values (Table 1). The intermittent group developed a thicker RV wall $(5.85 \mathrm{~mm} \pm 1.32 \mathrm{~mm})$ than the continuous group did $(4.54 \mathrm{~mm} \pm 0.51 \mathrm{~mm})$ at 96 hours $(P<.001)$. At the end of the training protocol, RV wall thickness increased $103.8 \%$ in the intermittent group and only $38.4 \%$ in the continuous group. No significant differences occurred in septal and LV wall thicknesses among groups $(P=.491)$ and across time $(P=.865)$. The graph
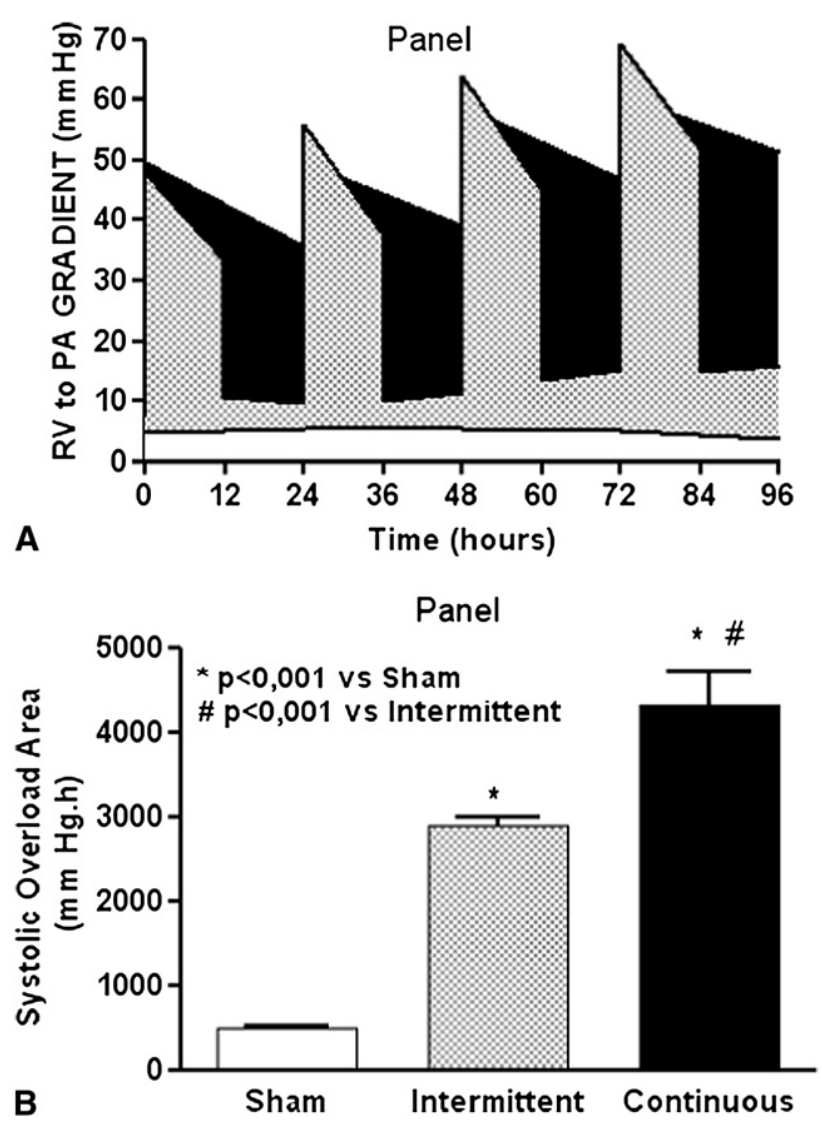

FIGURE 1. A, RV-PA gradient ( $\mathrm{mm} \mathrm{Hg}$ ) temporal pattern of sham, continuous, and intermittent groups. B, According to variations in RV-PA gradients in relation to time, the area under the curve $(\mathrm{mm} \mathrm{Hg} \mathrm{h}$ ) was calculated to determine the systolic overload imposed on each group. $R V$, Right ventricle; $P A$, pulmonary artery. $* P<.001$ compared with that of the sham group. $\# P<.001$ compared with the intermittent group.

with the percentage of changes in RV wall thickness is available online (Figure E3).

Regarding RV end-diastolic volume, there was a significant difference among groups $(P<.001)$ and throughout the protocol $(P=.024)$. RV volume was significantly dilated in the continuous group at 24 hours and thereafter, compared with that in the intermittent and sham groups

TABLE 1. RV wall thickness of the 3 groups measured by echocardiography in each time period of the protocol

\begin{tabular}{lccc}
\hline & \multicolumn{3}{c}{ RV wall thickness $(\mathbf{m m})$} \\
\cline { 2 - 4 } Time (h) & Sham $(\mathbf{n}=7)$ & Continuous $(\mathbf{n}=\mathbf{9})$ & Intermittent $(\mathbf{n}=\mathbf{1 1})$ \\
\hline Preop & $3.24 \pm 0.14$ & $3.28 \pm 0.35$ & $2.87 \pm 0.58$ \\
0 & $3.30 \pm 0.15$ & $3.31 \pm 0.33$ & $2.98 \pm 0.51$ \\
24 & $3.36 \pm 0.11$ & $3.37 \pm 0.33$ & $3.02 \pm 0.59$ \\
48 & $3.39 \pm 0.09$ & $3.63 \pm 0.68$ & $3.91 \pm 0.81^{*}$ \\
72 & $3.37 \pm 0.14$ & $4.40 \pm 0.64^{*}, \dagger$ & $4.85 \pm 0.66^{*}, \dagger$ \\
96 & $3.36 \pm 0.08$ & $4.54 \pm 0.51^{*}, \dagger$ & $5.85 \pm 1.32^{*}, \dagger, \dagger$ \\
\hline
\end{tabular}

Values $(\mathrm{mm})=$ means \pm standard deviation. $* P<.001$ compared with its respective baseline value. $\dagger P<.001$ compared with that in the sham group at the same time. $\ddagger P<.001$ compared with that in the continuous group at the same time. 


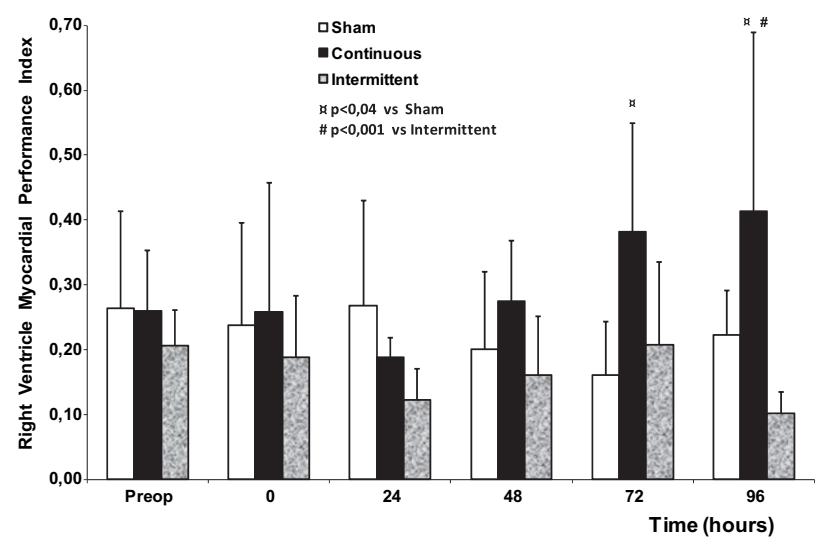

FIGURE 2. RV myocardial performance index during the 96-hour protocol in sham, continuous, and intermittent groups. $R V$, Right ventricle. $a P<.04$ compared with that in the sham group at the same time. $\# P<.001$ compared with that in the intermittent group at the same time.

$(P<.001)$. The graph with the percentage of changes in $\mathrm{RV}$ end-diastolic volume is available online (Figure E4). Worsening of RV myocardial performance index was observed in the continuous group at 72 hours and 96 hours of the protocol, compared with that in the sham group $(P<.039)$ and the intermittent group $(P<.001)$ at the end of the protocol (Figure 2).

\section{Morphologic Variables}

As demonstrated in Figure 3, the intermittent group had a $92.1 \%$ increase in $\mathrm{RV}$ mass $(1.46 \pm 0.53 \mathrm{~g} / \mathrm{kg})$ and a $46.5 \%$ increase in ventricular septal mass $(1.26 \pm 0.29$ $\mathrm{g} / \mathrm{kg})$ compared with the RV $(0.76 \pm 0.12 \mathrm{~g} / \mathrm{kg})$ and ventricular septal $(0.86 \pm 0.10 \mathrm{~g} / \mathrm{kg})$ masses in the sham group $(P<.05)$. On the other hand, the continuous group had an increase only in the ventricular septal mass $(1.18 \pm 0.14$ $\mathrm{g} / \mathrm{kg} ; P<.05)$. LV mass was not altered by RV training protocols $(P=.217)$. Regarding water content, both RV $(81.59 \% \pm 1.07 \%)$ and ventricular septum $(79.69 \% \pm$ $0.62 \%)$ in the continuous group and both RV $(81.84 \% \pm$ $1.11 \%)$ and ventricular septum $(79.45 \% \pm 0.62 \%)$ in the intermittent group had a mild, however significant, increase compared with that in the sham group (RV, 78.84\% \pm $2.41 \%$; ventricular septum, $77.11 \% \pm 2.08 \% ; P<.01$ ). No significant changes were observed in LV water content in the 3 groups.

\section{G6PD Activity}

G6PD activity ratio of $\mathrm{RV}$ to $\mathrm{LV}$ was significantly elevated by $130.1 \%$ in the continuous group $(P=.012)$, whereas the intermittent group showed a nonsignificant smaller increase of $39.8 \%$ in the G6PD activity ratio of $\mathrm{RV}$ to $\mathrm{LV}(P=.764)$ compared with that in the sham group (Figure 4$)$. There was no significant difference in the ventricular septum to LV G6PD activity ratio among groups $(P=.198)$.

\section{DISCUSSION}

The present experimental study aimed to compare PABinduced RV hypertrophy in continuous versus intermittent systolic overload in young goats, with the emphasis on G6PD activity analysis. Both study groups were capable of promoting different degrees of myocardial hypertrophy compared with the sham group. However, the intermittent group had greater RV and ventricular septal masses than the continuous group had, despite less exposure to systolic overload. The mild increase in RV and ventricular septum water content in both trained groups would not by itself justify the previous findings, suggesting that it was probably related to the enhanced protein synthesis and cell proliferation, as previously documented by Abduch and colleagues. $^{10}$ The continuous group had a series of deleterious effects at the end of the protocol. Persistent RV dilation was followed by impaired RV function and increased G6PD activity, G6PD being the rate-limiting enzyme in the oxidative branch of the pentose phosphate pathway. Because the pentose phosphate pathway is one of the major sources of NADPH in cardiac myocytes, this is an important finding, and it may indicate an unbalanced redox, with the occurrence of oxidative stress and generation of reactive oxygen species related to NADPH oxidase. ${ }^{11}$ In pathologic conditions, the excessive production of NADPH via G6PD overexpression is a result of multiple
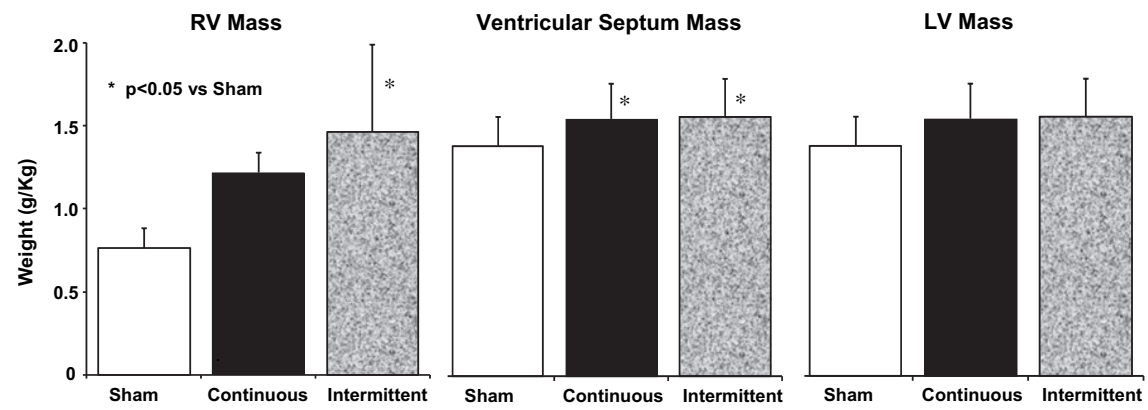

FIGURE 3. RV, ventricular septum, and LV weights in the 3 groups, indexed to body weight. Values $(\mathrm{g} / \mathrm{kg})=\operatorname{mean} \pm$ standard deviation. $R V$, Right ventricle; $L V$, left ventricle. $* P<.05$ compared with that in the sham group. 


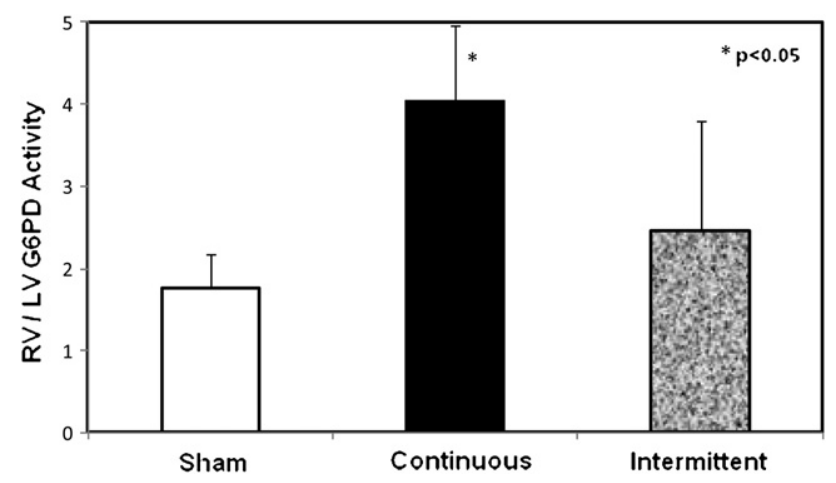

FIGURE 4. RV/LV ratio of G6PD activity in the sham, continuous, and intermittent groups. Values $=$ mean \pm standard deviation. Sham: $\mathrm{n}=5$; continuous: $\mathrm{n}=5$; intermittent: $\mathrm{n}=10 . R V$, Right ventricle; $L V$, left ventricle; $G 6 P D$, glucose 6-phosphate dehydrogenase. ${ }^{*} P<.05$ compared with that in the sham and intermittent groups.

factors, such as angiotensin II, thrombin, and tumor necrosis factor alpha. The final consequence of this cascade of events would be the cardiomyopathy related to protein aggregation owing to reductive stress. ${ }^{12,13}$

It has been demonstrated that either G6PD activation or inhibition is associated with diseases. However, growing evidence has emerged that G6PD overexpression correlates with oxidative and reductive stress, and new investigational drugs are currently under development to suppress its action. ${ }^{14}$ For instance, diabetic patients had upregulation of G6PD with high NADPH levels, and that was linked to inhibition of nitric oxide synthesis and endothelial dysfunction. ${ }^{15}$ Although the mechanisms underlying the increased production of reactive oxygen species in the heart are not completely understood, it has been proposed that the high rate of glucose oxidation increases mitochondrial membrane potential, which enhances production of superoxide anion. ${ }^{16,17}$ The latter would be a modulator in diabetic vasculopathy and precede the development of diabetic cardiomyopathy. ${ }^{18,19}$

Furthermore, in pacing-induced heart failure, an established model of dilated cardiomyopathy in dogs, it has been demonstrated that a 10-fold overexpression of G6PD occurs compared with that in controls. ${ }^{20}$ Recent studies demonstrated that G6PD upregulation in mice adipocytes has been implicated as one of the causes of altered hormonal release observed in obesity and insulin resistance. ${ }^{21}$

Although the present work has not evaluated the generation of reactive oxygen species related to NADPH oxidase, it is tempting to speculate that, in case of persistent systolic overload, upregulation and hyperactivity of myocardial G6PD observed in the continuous group strongly suggest that the pentose phosphate pathway enhances cytosolic NADPH availability, thus fueling free radical production by NADPH oxidase and uncoupled nitric oxide synthase. Therefore, it may induce superoxide anion myocardial injury, as well as protein aggregation, and subsequently heart failure. However, other unknown mechanisms of G6PD in heart failure could not be ruled out. This issue has been investigated worldwide.

Regarding the training protocol, previous studies have demonstrated that ventricular training protocols induce myocardial hypertrophy 96 hours after progressive systolic overload. $^{22}$ The search for a physiologic hypertrophy has been the focus of our laboratory for over 2 decades, and we postulate that intermittent systolic overload promotes the desirable effects of myocardial hypertrophy without its adverse effects. ${ }^{7}$ RV-PA gradients were more pronounced in the intermittent group after 48 hours of training. We would argue that the 12-hour resting period allowed the myocardium to replenish energy substrates and reestablish subendocardial perfusion owing to a lower ventricular wall tension. That would probably provide better hemodynamic performance at periods of systolic overload. ${ }^{23}$

\section{Study Limitations}

First, inferences based on animal findings do not necessarily translate into the same conclusions in humans. Second, RV hypertrophy was studied here, as opposed to human hearts with transposition of the great arteries. However, experimental models of aortic banding are associated with prohibited mortality rates. ${ }^{24}$ It is difficult to make definitive conclusions about a hypertrophic process based on a single enzyme activity. Nevertheless, it is essential to correlate these biochemical findings with production of superoxide anions and apoptosis to better understand the role of oxidative stress in hypertrophy training protocols. Previous studies have demonstrated that the oxidative branch of the pentose phosphate pathway, which produces NADPH and ribulose 5-phosphate, is essentially irreversible, being controlled primarily by G6PD activity and, hence, the NADPH/NADP ratio. $^{25}$ NADPH oxidase preferentially uses NADPH derived from the pentose phosphate pathway, and that, in the failing heart, more glucose is oxidized through the pentose phosphate pathway, with a consequent increase in electron donors available to fuel $\mathrm{O}_{2}^{-}$generating enzymes. Maybe that is the way $\mathrm{NADP}^{+}$is upregulated. Although this is a nonspecific pathway of free radical production, we have found a concordance of impaired RV function of continuous group and increased G6PD. Nevertheless, it would be more objective if we had measured oxygen-derived free radicals or tissue injury markers related to their production. Therefore, it is somehow difficult to assume and interpret a whole metabolic pathway based on the activity of a single enzyme. Future studies are being planned in our laboratory for the detection of increased oxidized glutathione, oxygen-derived free radicals, and myocardial injury markers that further supports the presence of a state of oxidative stress in the RV of the continuous group. 


\section{CONCLUSIONS}

This study demonstrates that continuous systolic overload for ventricle retraining causes hyperactivity in myocardial G6PD, together with RV dilation and dysfunction. That enzyme hyperactivity may be related to an unbalanced redox determined by a constant and pathologic systolic overload. Given that pentose phosphate pathway enhances cytosolic NADPH availability, this altered energy substrate metabolism can elevate levels of free radicals by NADPH oxidase, an important mechanism in the pathophysiology of heart failure. On the other hand, intermittent systolic overload has promoted a more efficient RV hypertrophy than the continuous one, with better preservation of myocardial performance and smaller G6PD activity. Clinical studies comparing adjustable and traditional pulmonary trunk banding should be welcomed to translate those findings to the practice of ventricular retraining.

We are grateful to Sachin A. Gupte, $\mathrm{MD}, \mathrm{PhD}$, from University of South Alabama, for his helpful suggestions on the revised manuscript.

\section{References}

1. Bishop SP, Melsen LR. Myocardial necrosis, fibrosis, and DNA synthesis in experimental cardiac hypertrophy induced by sudden pressure overload. Circ Res. 1976;39:238-45.

2. Huss JM, Kelly DP. Mitochondrial energy metabolism in heart failure: a question of balance. J Clin Invest. 2005;115:547-55.

3. Gupte RS, Vijay V, Marks B, Levine RJ, Sabbah HN, Wolin MS, et al. Upregulation of glucose-6-phosphate dehydrogenase and NAD(P)H oxidase activity increases oxidative stress in failing human heart. J Card Fail. 2007;13:497-506.

4. Luzzato L, Battistuzzi G. Glucose-6-phosphate dehydrogenase. Adv Hum Genet. 1985; $14: 217-329$

5. Gupte SA, Levine RJ, Gupte RS, Young ME, Lionetti V, Labinskyy V, et al. Glucose-6-phosphate dehydrogenase-derived NADPH fuels superoxide production in the failing heart. J Mol Cell Cardiol. 2006;41:340-9.

6. Iemitsu M, Miyauchi T, Maeda S, Sakai S, Fujii N, Miyazaki H, et al. Cardiac hypertrophy by hypertension and exercise training exhibits different gene expression of enzymes in energy metabolism. Hypertens Res. 2003;26:829-37.

7. Valente AS, Assad RS, Abduch MC, Silva GJ, Thomaz PG, Miana LA, et al. Pulmonary trunk reversible banding. IV: analysis of right ventricle acute hypertrophy in an intermittent overloading experimental model. Braz J Cardiovasc Surg. 2008;23:60-9.

8. Fulton RM, Hutchinson EC, Jones AM. Ventricular weight in cardiac hypertrophy. Br Heart J. 1952;14:413-20.

9. Bergmeyer HU, Bernt E. D-Glucose determination with hexokinase and glucose6-phosphate dehydrogenase. In: Bergmeyer HU, ed. Methods of enzymatic analysis. London: Academic Press; 1974. p. 1196-201.

10. Abduch MC, Assad RS, Rodriguez MQ, Valente AS, Andrade JL, Demarchi LM, et al. Reversible pulmonary trunk banding III: assessment of myocardial adaptive mechanisms-contribution of cell proliferation. J Thorac Cardiovasc Surg. 2007; 133:1510-6.

11. Rajasekaran NS, Connell P, Christians ES, Yan LJ, Taylor RP, Orosz A, et al. Human alpha B-crystallin mutation causes oxido-reductive stress and protein aggregation cardiomyopathy in mice. Cell. 2007;130:427-39.

12. Zimmer HG. Regulation of and intervention into the oxidative pentose phosphate pathway and adenine nucleotide metabolism in the heart. Mol Cell Biochem. 1996;160:101-9.

13. Matsui R, Xu S, Maitland KA, Hayes A, Leopold JA, Handy DE, et al. Glucose 6phosphate dehydrogenase deficiency decreases the vascular response to angiotensin II. Circulation. 2005;112:257-63.

14. Gupte SA. Glucose-6-phosphate dehydrogenase: a novel therapeutic target in cardiovascular diseases. Curr Opin Invest Drugs. 2008;9:993-1000.

15. Guzik TJ, Mussa S, Gastaldi D, Sadowski J, Ratnatunga C, Pillai R, et al. Mechanisms of increased vascular superoxide production in human diabetes mellitus: role of NADPH oxidase and endothelial nitric oxidase synthase. Circulation. 2002; 105:1656-62.

16. An D, Rodrigues B. Role of changes in cardiac metabolism in development of diabetic cardiomyopathy. Am J Physiol Heart Circ Physiol. 2006;291:H1489-506.

17. Hink U, Li H, Mollnau H, Oelze M, Matheis E, Hartmann M, et al. Mechanisms underlying endothelial dysfunction in diabetes mellitus. Circ Res. 2001;88:E14-22.

18. Park J, Rho HK, Kim KH, Choe SS, Lee YS, Kim JB. Overexpression of glucose 6-phosphate dehydrogenase is associated with lipid dysregulation and insulin resistance in obesity. Moll Cell Biol. 2005;25:5146-57.

19. Serpillon S, Floyd BC, Gupte RS, George S, Kozicky M, Neito V, et al. Superoxide production by $\mathrm{NAD}(\mathrm{P}) \mathrm{H}$ oxidase and mitochondria is increased in genetically obese and hyperglycemic rat heart and aorta before the development of cardiac dysfunction. The role of glucose-6-phosphate dehydrogenase-derived NADPH Am J Physiol Heart Circ Physiol. 2009;297:H153-62.

20. Recchia FA, McConnell PI, Bernstein RD, Vogel TR, Xu X, Hintze TH. Reduced nitric oxide production and altered myocardial metabolism during the decompensation of pacing-induced heart failure in the conscious dog. Circ Res. 1998;83: 969-79.

21. Lopaschuk GD, Folmes CD, Stanley WC. Cardiac energy metabolism in obesity. Circ Res. 2007; 101:335-47.

22. Dias CA, Assad RS, Caneo LF, Abduch MC, Aiello VD, Dias AR, et al. Reversible pulmonary trunk banding. II. An experimental model for rapid pulmonary ventricular hypertrophy. J Thorac Cardiovasc Surg. 2002;124:999-1006.

23. Miana LA, Assad RS, Abduch MC, Gomes GS, Nogueira AR, Oliveira FS, et al Intermittent systolic overload promotes better myocardial performance in adul animals. Arq Bras Cardiol. 2010;95:364-72

24. Bonnet D, Maillard A, Chetboul V, Pouchelon JL, Aggoun Y, Acar P, et al Préparation non chirurgicale du ventricle sous-pulmonaire à la détransposition artérielle: mise au point d'um modèle animal chez l'agneau. Arch Mal Coeur Vaiss. 1997;90:707-12.

25. Leong HS, Grist M, Parsons H, Wambolt RB, Lopaschuk GD, Brownsey R, et al Accelerated rates of glycolysis in the hypertrophied heart: are they a methodological artifact? Am J Physiol Endocrinol Metab. 2002;282:E1039-45.

\section{Discussion}

Dr William J. Brawn (Birmingham, United Kingdom). I have no disclosures. I have 2 questions.

In the human situation, acutely raising the ventricular ratio to $70 \%$ is usually followed by a fall in this ratio, probably with an associated falling cardiac output. How do you adjust the band, and how frequently, to maintain that $70 \%$ ratio in your animals?

Dr Atik. That is a very good point. Thank you for your question. In our study, we performed the operation, and we have allowed the heart to recover for 72 hours before we initiated training. By the time we did it, we tightened the band to achieve that ratio. Sometimes we have to loosen it a little bit because there was a drop of more than $10 \%$ in systolic blood pressure or there were arrhythmias or agitation and hypoxia. So we went back to 0.5 or 0.6 ratio and waited a few minutes and then we tried it again. If it was not possible, we left the band tightened up to a tolerable point for the animal. Reassessments were made every 24 hours and in almost all the animals we had to readjust at that time.

Dr Brawn. Thank you. Final question, have you any insight as to how long it takes to upregulate the G6PD activity, how acutely it occurs?

Dr Atik. We do not have an answer for that question. This is a very good point. Actually, G6PD activity has a 2-fold mechanism. First, it enhances ribose 6-phosphate, which is a precursor of de novo production of DNA, which is good for hypertrophy. On the other hand, G6PD may enhance superoxide anion generation, which is bad for hypertrophy and can induce myocardial injury. So we really do not know how long does it take to modulate its activity. 


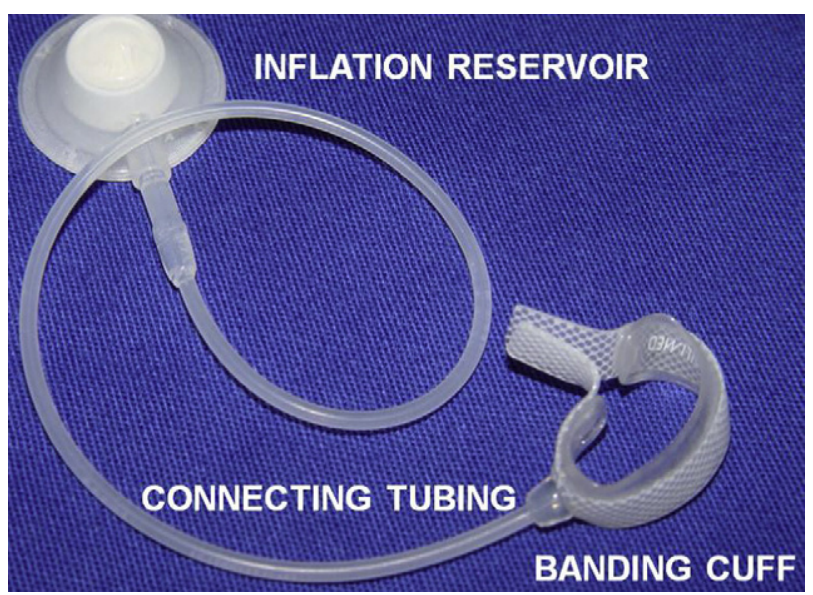

FIGURE E1. Pulmonary trunk adjustable banding device used in the protocol, manufactured by SILIMED, Silicone e Instrumental Médico-Cirúrgico e Hospitalar Ltda, Rio de Janeiro, Brazil.

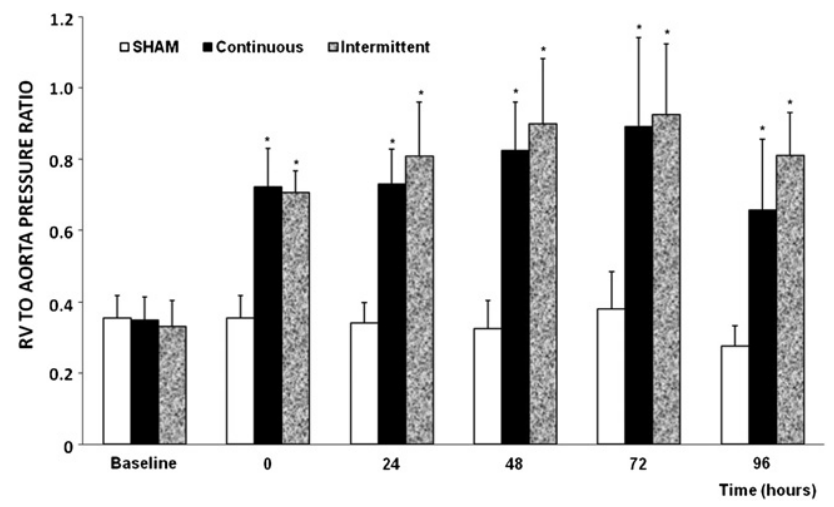

FIGURE E2. RV/aorta systolic pressure ratio of sham, continuous, and intermittent groups, throughout the protocol. $R V$, Right ventricle. ${ }^{*} P<.05$ compared with its respective baseline and with that in the sham group at the same time.

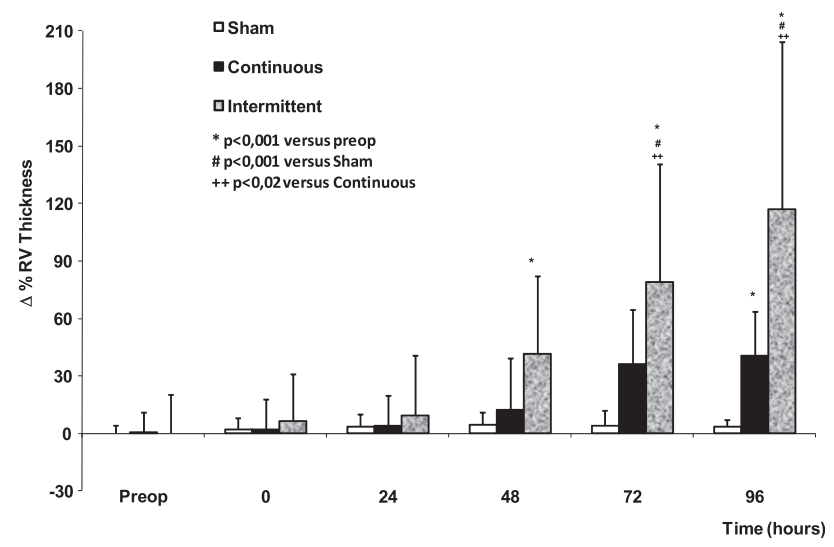

FIGURE E3. $\Delta \%$ changes of RV wall thickness of sham, continuous, and intermittent groups throughout the protocol. $R V$, Right ventricle. $* P<.001$ compared with the respective baseline value. $\# P<.001$ compared with the sham group at the same time. $+P>.02$ compared with the continuous group at the same time.

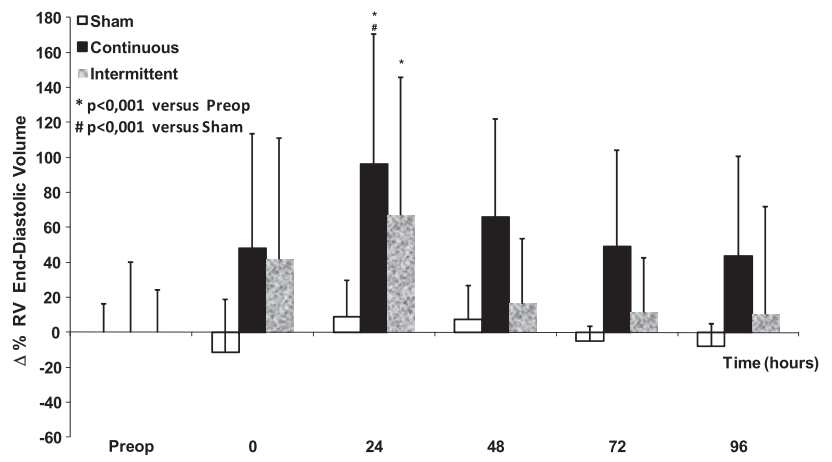

FIGURE E4. $\Delta \%$ changes of RV end-diastolic volume of sham, continuous, and intermittent groups throughout the protocol. $R V$, Right ventricle. $* P<.001$ compared with the respective baseline value. $\# P<.001$ compared with the sham group at the same time.

TABLE E1. RV-PA systolic pressure gradients of the 3 groups in each time period of the protocol

\begin{tabular}{lcccc}
\hline & \multicolumn{3}{c}{ RV-PA gradient $(\mathbf{m m}$ Hg) } & \\
\cline { 2 - 4 } Time (h) & Sham & Continuous & Intermittent & P value* \\
\hline Baseline & $5.43 \pm 1.99$ & $8.00 \pm 3.79$ & $7.73 \pm 6.40$ & 1.00 \\
0 & $5.43 \pm 1.99$ & $49.63 \pm 16.70$ & $48.00 \pm 10.93$ & 1.00 \\
24 & $5.43 \pm 1.27$ & $49.43 \pm 14.37$ & $55.73 \pm 9.91$ & .69 \\
48 & $5.43 \pm 1.13$ & $58.88 \pm 11.43$ & $63.82 \pm 13.69$ & .97 \\
72 & $5.50 \pm 1.38$ & $60.88 \pm 15.72$ & $69.18 \pm 9.78$ & .30 \\
96 & $3.83 \pm 1.33$ & $51.29 \pm 21.47$ & $66.20 \pm 11.37$ & .06 \\
\hline
\end{tabular}

Values $=$ mean \pm standard deviation. $R V$, Right ventricle; $P A$, pulmonary artery. $* P$ value of comparisons for continuous $\times$ intermittent groups. 